\title{
Sistema de pré-aviso para controle de Sigatoka-amarela no norte de Minas Gerais
}

\author{
Sara de Almeida Rios ${ }^{1 *}$ \\ Mário Sérgio Carvalho Dias ${ }^{2}$ \\ Zilton José Maciel Cordeiro ${ }^{3}$ \\ Wagner Martins de Souza ${ }^{4}$ \\ João José Costa Silva ${ }^{5}$ \\ José Aparecido Alves Barbosa ${ }^{6}$ \\ Renata Silva Canuto de Pinho ${ }^{7}$ \\ Samuel Campos Abreu ${ }^{8}$ \\ Leandra Oliveira Santos ${ }^{9}$ \\ ${ }^{1}$ Embrapa Amazônia Ocidental \\ Rodovia AM-010, Km 29, Zona Rural, Caixa Postal 319, CEP 69010-970, Manaus - AM, Brasil \\ ${ }^{2}$ EPAMIG/ Núcleo Tecnológico EPAMIG Batata e Morango \\ ${ }^{3}$ Embrapa Mandioca e Fruticultura, Cruz das Almas - BA, Brasil \\ ${ }^{4}$ Universidade Estadual de Montes Claros, Montes Claros - MG, Brasil \\ ${ }^{5}$ Universidade Estadual de Roraima, Rorainopolis - RR, Brasil \\ ${ }^{6}$ SEMAD - Secretaria de Estado de Meio Ambiente e Desenvolvimento Sustentável \\ ${ }^{7}$ Universidade Federal do Pampa, Itaqui - RS, Brasil \\ ${ }^{8}$ Embrapa Produtos e Mercado \\ ${ }^{9}$ EPAMIG/CTNM - Centro Tecnológico do Norte de Minas \\ * Autor para correspondência \\ sara.rios@embrapa.br
}

Submetido em 02/10/2012

Aceito para publicação em 28/03/2013

\section{Resumo}

O objetivo do presente trabalho foi avaliar o Sistema de Pré-Aviso Biológico (SPB) para o controle da Sigatoka-amarela no Norte de Minas. O ensaio foi conduzido no município de Nova Porteirinha, MG. Foram testados sete tratamentos, utilizando seis valores de soma bruta (SB) $(1.000 ; 1.300 ; 1.600 ; 1.900 ; 2.200$ e 2.500) e o controle sistemático da doença a cada 15 dias. Os tratamentos foram distribuídos em sete talhões (120 plantas/talhão) com 'Prata-Anã' e 10 plantas de cada talhão foram avaliadas semanalmente quanto à taxa de emissão foliar e incidência da doença nas folhas dois, três e quatro. Os estádios mais avançados das lesões e a sua intensidade serviram para cálculo da soma bruta, que auxiliou na tomada de decisão para o controle químico. Foram coletados os dados de produção e análises de firmeza, $\mathrm{pH}$ e acidez dos frutos. Para o tratamento SB 2.500, considerando os dois anos de avaliação, houve uma redução de doze para três aplicações, ou seja, $75 \%$ menos defensivos aplicados, sem perda na produtividade. Logo, sugere-se, considerando as condições de Nova Porteirinha, a aplicação do SPB para o controle químico da Sigatoka-amarela, utilizando-se o valor de SB de 2.500 como indicador da época correta para a realização da pulverização.

Palavras-chave: Banana; Fungicidas; Musa sp.; Mycosphaerella musicola; Sistema de pré-aviso 


\section{Abstract}

Forewarning system for controlling Yellow Sigatoka in Northern Minas Gerais, Brazil. This trial aimed to evaluate the Biological Forewarning System (BFS) for controlling Yellow Sigatoka in Northern Minas Gerais, Brazil. It was carried out in the town of Nova Porteirinha. One tested 7 treatments, using 6 gross sum (GS) values $(1,000 ; 1,300 ; 1,600 ; 1,900 ; 2,200$, and 2,500) and the systematic disease control every 15 days. The treatments were distributed into 7 plots (120 plants/plot) with 'Prata-Anã' and 10 plants from each plot were weekly evaluated with regard to leaf emission rate and disease incidence on the leaves 2, 3 and 4 . The more advanced lesion stages and their intensity were used to calculate the gross sum, which aided in decision-making for chemical control. One collected data on production and firmness, $\mathrm{pH}$, and acidity analysis of fruits. For the GS 2,500 treatment, taking into account 2-year evaluation, there was a reduction from 12 to 3 applications, i.e. $75 \%$ less fungicide was applied, without loss in productivity. Therefore, one suggests, taking into account the conditions of Nova Porteirinha, the application of BFS for chemical control of Yellow Sigatoka, using the GS value of 2,500 as an indicator of the correct time to perform pulverization.

Key words: Banana; Forewarning system; Fungicides; Musa sp.; Mycosphaerella musicola

\section{Introdução}

A bananicultura é uma atividade agrícola de grande relevância para o Brasil, totalizando 7.119.940 toneladas, distribuídas nos 514.366 ha (IBGE, 2012). Entre os estados produtores, Minas Gerais se destaca pela posição geográfica e condições edafoclimáticas, sendo a banana cultivada em todo o estado. No Norte de Minas, o cultivo da banana 'Prata-Anã' concentra cerca de 12.000 ha, tornando-se um dos pólos de maior concentração de produção no país (ABANORTE, 2005; MDIC, 2005; TODA FRUTA, 2006).

Dentre os desafios da bananicultura está o controle da Sigatoka-amarela, doença causada pelo fungo Mycosphaerella musicola Leach (= Pseudocercospora musae (Zimm) Deighton), podendo causar perdas superiores a $50 \%$ da produção, se não controlada.

Apesar das condições de clima seco, chuvas escassas e baixa umidade, características da região norte do estado de Minas Gerais, verifica-se o desenvolvimento da doença nesta região, principalmente pelos microclimas presentes na bananicultura irrigada, os quais poderiam ser amenizados integrando-se os controles culturais, químicos, biológicos e genéticos, excetuando-se este último para o grupo "Prata-Anã", sem cultivares resistentes à Sigatoka-amarela. No entanto, no Norte de Minas, dentre os diferentes métodos de controle da doença, prevalece o uso sistemático e indiscriminado de produtos químicos. A consequência é um elevado custo de produção, efeitos prejudiciais ao meio ambiente além da pressão de seleção sobre o patógeno, aumentando os riscos da seleção de variantes insensíveis aos fungicidas mais empregados. Neste sentido, linhas de pesquisa, como o sistema de pré-aviso biológico (SPB), vêm sendo avaliadas com o intuito de se estabelecer um mecanismo de controle que seja mais eficiente ao tradicional calendário de aplicações de fungicidas empregado pelos bananicultores do Norte de Minas.

O SPB prevê a taxa de desenvolvimento da doença por meio de escalas de sintomas vistos nas folhas da bananeira. Conforme Ferreira et al. (2003), o método de previsão se baseia na interação entre o estádio de desenvolvimento da folha vela e a severidade da doença nas folhas mais novas. Este sistema leva em consideração um valor de soma bruta (SB) que quantifica a severidade da doença, indicando o melhor momento para realização do controle químico, logo, com maior probabilidade de racionalização do uso de fungicidas.

Alguns sistemas de pré-aviso biológicos foram inicialmente propostos pelos franceses e, posteriormente, adaptados às condições da Costa Rica (GANRY; MEYER, 1972; CORDEIRO, 2000). No Brasil, Ferreira et al. (2003) estudaram o SPB no Recôncavo baiano, com redução de $40 \%$ no número de aplicações de fungicidas, utilizadas em intervalos pré estabelecidos de 21 dias. No entanto, uma das premissas básicas para o sucesso no controle da Sigatoka-amarela é a realização de estudos localizados, a fim de ajustar qualquer que seja o método, às condições da região. Portanto, o objetivo do presente 
trabalho foi avaliar o SPB para o controle da Sigatokaamarela na região de Nova Porteirinha, norte de Minas Gerais.

\section{Material e Métodos}

O experimento foi conduzido em área experimental pertencente à Epamig - Centro Tecnológico do Norte de Minas, localizada no município de Nova Porteirinha, Minas Gerais. Utilizou-se a 'Prata-Anã' (grupo genômico $\mathrm{AAB}$ ), suscetível à Sigatoka-amarela, no sistema de plantio em fileiras duplas, no espaçamento $4 \mathrm{~m} \times 2 \mathrm{~m} \times 2 \mathrm{~m}$. Foram realizados sete tratamentos: 1. Controle sistemático (CS), com aplicação de fungicida a cada 15 dias, aproximadamente; 2. SB 1000; 3. SB 1300; 4. SB 1600; 5. SB 1900; 6. SB 2200 e 7. SB 2500 , sendo que cada tratamento foi constituído por 120 plantas, avaliando-se dez plantas aleatórias de cada tratamento.

A coleta de dados foi semanal, registrando-se em uma ficha a taxa de emissão foliar (estádio de evolução da folha vela), segundo escala proposta por Brun (1963) e a incidência da doença, nas folhas 2, 3 e 4, segundo a escala proposta por Fouré (1994). Além da incidência, avaliou-se a densidade de ataque observada, tomando-se como referência o número de 50 lesões no nível mais elevado de desenvolvimento (nas folhas 2, 3 e 4). Para isso, empregou-se um sinal de mais $(+)$ quando observadas mais de 50 lesões no estádio mais desenvolvido ou sinal de menos (-) quando o número de lesões neste estádio era menor que 50 .

Os dados coletados foram utilizados para o monitoramento da severidade da Sigatoka-amarela, utilizando-se o método de Estado de Evolução (EE), baseado no sistema de pré-aviso biológico desenvolvido por Ganry e Meyer (1972). Este método considera o ritmo de emissão foliar semanal (REF) e os estádios precoces do desenvolvimento dos sintomas (1, 2 e 3$)$ presentes na segunda (II), terceira (III) e quarta (IV) folha, a partir da "vela. O estado da evolução da severidade foi definido pela equação: $\mathrm{EE}=\mathrm{SEV}$ x REFx; onde SEV é severidade semanal da doença e REFx, o ritmo de emissão foliar (taxa de emissão foliar) ponderado, sendo este, a média entre o ritmo de emissão foliar da semana passada
(REFp) e a atual (REFa) de avaliação. Para o cálculo do valor de soma bruta atingido, os dados referentes ao estádio da lesão e densidade de ataque nas folhas 2, 3 e 4 foram multiplicados pelos coeficientes estabelecidos por Ganry e Laville (1983).

As avaliações foram realizadas semanalmente de outubro de 2003 a março de 2005 e os cálculos de REF, $\mathrm{SB}$ atingida e EE da doença foram obtidos por meio do software cedido pela Corporación Bananera Nacional (Corbana), da Costa Rica. O valor de SB atingido era a referência para a indicação do controle químico, para cada tratamento, ou seja, implementou-se o controle químico da doença sempre que a doença atingiu os limites de SB preestabelecidos para cada tratamento. Este controle químico foi realizado utilizando-se atomizador costal motorizado modelo MK 30, com capacidade para $20 \mathrm{~L}$, fazendo-se a alternância de princípios ativos apresentados na Tabela 1 .

Os tratos culturais utilizados na condução do experimento foram aqueles em uso na bananicultura. A colheita foi realizada 12 meses após a instalação do sistema de pré-aviso. Foram avaliados: massa dos cachos, número de pencas e caracterização físico-química dos frutos. As avaliações de resistência da polpa, utilizandose um penetrômetro (marca FERRARI) foram feitas em unidade experimental composta de três frutos, oriundos da segunda penca. As análises químicas constaram das seguintes variáveis: $\mathrm{pH}$, medido por potenciômetro TECNAL modelo pH Meter TEC-2 e sólidos solúveis (SS), determinados em refratômetro digital modelo $\mathrm{N}-1 \mathrm{E}$ (Atago Co., LTD., Japão), expressando-se os resultados em porcentagem conforme normas da AOAC (1990).

TABELA 1: Produtos químicos e suas respectivas dosagens para o controle da Sigatoka-amarela em bananeiras 'Prata-Anã' em sistema de PréAviso Biológico, no norte de Minas Gerais. Nova Porteirinha, MG, 2005.

\begin{tabular}{lc}
\hline \multicolumn{1}{c}{ Produtos } & Dosagem de i.a./ha \\
\hline Óleo mineral & 12 a $15 \mathrm{~L}$ \\
Propiconazol + óleo mineral & 100 a $125 \mathrm{~mL}$ \\
Thiabendazol + óleo mineral & 125 a $150 \mathrm{~mL}$ \\
Azoxistrobina & 125 a $150 \mathrm{~mL}$ \\
\hline
\end{tabular}


O delineamento estatístico utilizado foi o inteiramente casualizado, com sete tratamentos e 10 repetições (análises de produção) e sete tratamentos e cinco repetições de três frutos na parcela (análises pós-colheita). Os dados foram submetidos à análise de variância e as médias foram comparadas pelo Teste de Tukey ao nível de 5\% de probabilidade.

\section{Resultados e Discussão}

Os fatores umidade e temperatura apresentam-se extremamente importantes para a epidemiologia da Sigatoka-amarela. As curvas de progresso da doença (Figura 1) mostram efeito sazonal relevante, com severidade mais elevada no período chuvoso e com temperaturas altas, correspondente aos meses de outubro a março e com níveis decrescentes entre os meses de maio e setembro, período menos chuvoso e mais frio. Resultados semelhantes foram encontrados por Ferreira et al. (2003) avaliando o sistema de pré-aviso em bananeira no Recôncavo Baiano.
A severidade da doença foi proporcional ao rigor na aplicação de fungicidas, ou seja, quanto maior a frequência na aplicação dos fungicidas, menor a incidência da doença, considerando os limites de SB preestabelecidos (Figura 1; Tabela 2). Este resultado já era esperado pelo comportamento inversamente proporcional da severidade da doença e o rigor no controle químico (aplicação de fungicidas). Logo, verificou-se o menor índice de doença exatamente para o Controle Sistemático quinzenal (Figura 1). Além disso, padrão semelhante foi verificado para os tratamentos SB 1000 e SB 1300. Esta baixa amplitude de variação na evolução da Sigatoka-amarela pode ser devida à manutenção da doença em níveis baixos com a aplicação mais frequente de fungicidas (Tabela 2). No entanto, não devem ser priorizados os tratamentos que mantenham os menores índices da doença e sim aqueles que garantam a máxima produtividade com o mínimo de aplicações de fungicidas.

A curva de evolução da doença (Figura 1), para o tratamento controle sistemático, apresentou variações mais suaves em relação aos demais tratamentos, o que

FIGURA 1: Níveis de Sigatoka-amarela, representada pela média mensal da soma bruta (SB), mediante leitura do estádio de lesão da doença, entre outubro de 2003 e março de 2005, em bananeiras 'Prata-Anã' submetidas a sete tratamentos distintos (C. Sist. = controle sistemático da doença com aplicação quinzenal de fungicidas e seis valores de SB limites preestabelecidos), Nova Porteirinha, MG.




TABELA 2: Número total de aplicações de fungicidas e médias de massa dos cachos, número de pencas, firmeza, pH e SST dos frutos oriundos de bananeiras 'Prata-Anã' submetidas ao sistema de pré-aviso biológico para controle de Sigatoka-amarela entre outubro de 2003 a março de 2005. Nova Porteirinha, MG.

\begin{tabular}{lccccccc}
\hline \multicolumn{7}{c}{ Tratamentos* $^{*}$} \\
\hline Variáveis & $\mathbf{C S}^{\mathbf{1}}$ & $\mathbf{1 0 0 0}$ & $\mathbf{1 3 0 0}$ & $\mathbf{1 6 0 0}$ & $\mathbf{1 9 0 0}$ & $\mathbf{2 2 0 0}$ & $\mathbf{2 5 0 0}$ \\
\hline № de aplicações & 28 & 12 & 12 & 9 & 10 & 5 & 3 \\
Massa do cacho $(\mathrm{kg})$ & $13,4 \mathrm{~b}$ & $15,8 \mathrm{~b}$ & $20,6 \mathrm{ab}$ & $17,1 \mathrm{ab}$ & $13,7 \mathrm{~b}$ & $18,5 \mathrm{ab}$ & $24,7 \mathrm{a}$ \\
№ de pencas & $8,2 \mathrm{~b}$ & $9,6 \mathrm{ab}$ & $11,0 \mathrm{a}$ & $10,2 \mathrm{ab}$ & $9,0 \mathrm{ab}$ & $10,4 \mathrm{ab}$ & $10,6 \mathrm{a}$ \\
Firmeza & $7,4 \mathrm{~ns}$ & $6,7 \mathrm{~ns}$ & $6,3 \mathrm{~ns}$ & $6,7 \mathrm{~ns}$ & $6,6 \mathrm{~ns}$ & $7,0 \mathrm{~ns}$ & $6,5 \mathrm{~ns}$ \\
pH & $5,7 \mathrm{~ns}$ & $5,8 \mathrm{~ns}$ & $5,7 \mathrm{~ns}$ & $5,6 \mathrm{~ns}$ & $5,7 \mathrm{~ns}$ & $5,8 \mathrm{~ns}$ & $5,7 \mathrm{~ns}$ \\
SST ('brix) & $0,9 \mathrm{~ns}$ & $1,0 \mathrm{~ns}$ & $0,9 \mathrm{~ns}$ & $0,8 \mathrm{~ns}$ & $0,9 \mathrm{~ns}$ & $0,7 \mathrm{~ns}$ & $0,8 \mathrm{~ns}$ \\
\hline
\end{tabular}

*Valores de soma bruta (SB) preestabelecidos. ${ }^{1}$ Controle sistemático da doença com aplicação quinzenal de fungicidas. ns = não significativo pelo teste $\mathrm{F}$ ao nível de $5 \%$ de probabilidade. Médias seguidas da mesma letra na linha não diferem entre si, pelo teste Tukey ao nível de $5 \%$ de probabilidade.

é totalmente justificável pela frequência quinzenal de aplicação de fungicidas. No entanto, apesar de as curvas dos demais tratamentos terem apresentado variações mensais mais bruscas, em alguns pontos (meses) observa-se que os tratamentos SB 1000, SB 1300 e SB 1600 apresentaram valores de SB (evolução da doença) menores e/ou iguais ao tratamento controle sistemático. Resultados semelhantes foram verificados por Ferreira et al. (2003) os quais concluem que as aplicações de fungicidas, quando realizadas no momento correto, proporcionam maior eficiência no controle da Sigatokaamarela do que a aplicação sistemática de fungicidas pela prática do calendário fixo empregada pelos produtores, a qual não leva em consideração a avaliação da severidade da doença. Pérez (2000) corrobora com estes autores ao relatar que a eficiência está diretamente relacionada ao monitoramento da severidade da doença e indicação de aplicações químicas no momento adequado.

Na Tabela 2, podem ser verificados os valores médios para as características relacionadas à produtividade $\mathrm{e}$ qualidade físico-química das bananeiras, sendo que a massa do cacho foi superior para o controle SB 2500 $(24,7$ kg) em relação aos tratamentos CS, SB 1000 e SB 1900. Porém, estatisticamente igual àquela observada para os demais tratamentos (SB 2200, SB 1600 e SB 1300). Os menores valores médios de massa de cacho e número de pencas verificados no tratamento $\mathrm{CS}$ podem ser justificados pela alta frequência na aplicação de fungicidas, em especial, considerando a aplicação conjunta do fungicida em mistura com óleo mineral, este último podendo apresentar fitotoxicidade. Segundo FHIA (2004), altas doses de óleo mineral aplicadas em período mais secos e/ou em solos mal drenados podem resultar em elevada fitotoxicidade, refletindo na quantidade e qualidade de cachos produzidos. Novas metodologias de aplicação de fungicidas vêm sendo recomendadas na bananicultura, de forma a otimizar a eficiência do controle da Sigatoka-Amarela, a exemplo da aplicação localizada. Gasparotto et al. (2007) recomendam esta aplicação, via seringa, com inserção do produto direto na axila da folha, a qual diminui a introdução de defensivos agrícolas na cadeia trófica, evita o desperdício e diminui os riscos de contaminação ambiental. Cezar et al. (2002), injetando benomil e propiconazole no pseudocaule da cultivar Pacovan para o controle da Sigatoka-amarela, verificaram que os produtos foram eficientes, porém alertam sobre a necessidade de mais estudos para consolidar essa forma de aplicação. Apesar de muito promissora, a aplicação localizada está pouco difunda entre os bananicultores do Norte de Minas, talvez por falta de conhecimento, ou ainda, por ser uma metodologia mais apropriada e vantajosa aos pequenos produtores.

Dentre os tratamentos que obtiveram as maiores massas de cacho (SB 1300, SB 1600, SB 2200 e SB 2500), para os quais foram necessárias, respectivamente, 
12, 9, 5 e 3 aplicações de fungicidas durante todo o período de avaliação, observa-se que a maior eficiência foi verificada no SB 2500, com uma redução de 12 para apenas três aplicações químicas, sem perda na produção (Tabela 2). Estes resultados implicam em menor custo e maior preservação ambiental, ao reduzir o número de aplicações em 75\%. Há também redução da pressão de seleção sobre o patógeno, reduzindo os riscos de surgimento de variantes menos sensíveis aos fungicidas comumente empregados no controle da doença.

Apesar de Borges et al. (2009) relatarem como prejuízos da Sigatoka-amarela a maturação precoce dos frutos ainda no campo e durante o transporte póscolheita, não foram observadas diferenças estatísticas para as variáveis firmeza, $\mathrm{pH}$ e SST entre os tratamentos avaliados. Estes resultados já eram esperados visto que os principais distúrbios observados em plantios afetados pela doença estão relacionados aos caracteres diretos de produção, principalmente quanto à diminuição da produtividade, do número de pencas e do tamanho dos frutos (FIORAVANÇO; PAIVA, 2005).

Considerando esta como sendo a primeira avaliação de um sistema de pré-aviso biológico para a região do Norte de Minas, e, considerando ainda, que os resultados foram promissores quanto à produtividade e número de aplicações químicas, sugere-se que, no município de Nova Porteirinha, região norte do Estado de Minas Gerais, o controle químico da Sigatoka-amarela deve ser iniciado quando a Soma Bruta atingir o valor de 2500. Porém, é importante ressaltar que cada um dos diferentes métodos de aplicação de fungicidas empregados no controle da Sigatoka seja por aplicações aéreas (aviões ou helicópteros) ou terrestres (pulverizadores tratorizados, atomizadores costais e aplicações manuais via seringa na axila da folha) apresenta vantagens e desvantagens, principalmente quanto ao custo das aplicações, praticidade, eficiência de controle e agressão ao meio ambiente. Além disso, após o ingresso da "Sigatoka-negra" no Brasil, métodos de controle integrado da doença evoluíram bastante, como no caso de sistemas de controle por pré-aviso biológico adotados em algumas regiões do país (MORAES et al., 2010), ou ainda, dos sistemas de pré-aviso bioclimáticos. É preciso que estas avaliações sejam realizadas e validadas nas mais diversas regiões produtoras de banana do país de forma que a convivência com a Sigatoka-amarela seja viável economicamente.

\section{Referências}

ABANORTE. Desempenho da fruticultura mineira em 2004. 2005. Disponível em: <http://www.abanorte.com.br/fruticultura>. Acesso em: 29 dez. 2005.

AOAC - ASSOCIATION OF OFFICIAL AGRICULTURAL CHEMISTS. Official methods of the Association of the Agricultural Chemists. Vol. 2. 15 ed. Washington: AOAC, 1990. $1017 \mathrm{p}$.

BORGES, A. L.; SILVA, A. L. DA; BATISTA, D. DA C.; MOREIRA, F. R. B.; FLORI, J. E.; ARAUJO, J. L. P. Sistemas de produção da bananeira irrigada. Petrolina: Embrapa Semiárido, 2009. (Embrapa Semiárido. Sistemas de Produção, 4). Disponível em: <http://sistemasdeproducao.cnptia.embrapa.br/FontesHTML/ Banana/BananeiraIrrigada/index.htm>.

BRUN, J. La Cercosporiose du bananier in Guinée: etude de la phase Ascorporée du Mycosphaerella musicola Leach. 1963. 16. Tese (Doctor em Science) -IFAC, Paris, (Série A. Orsay, 35). 1963. CEZAR, J. O.; LEITE, J. B. V.; RAMOS, J. V.; PEREIRA, J. L. M.; FRIFE FILHO, G. A. Efeito da aplicação de fungicidas via injeção no controle da sigatoka-amarela (Mycosphaerella musicola Leach) na bananeira. In: CONGRESSO BRASILEIRO DE FRUTICULTURA, 17, 2002, Belém. Anais... Belém: Sociedade Brasileira de Fruticultura, 2002. Vol. 1. CD-ROM.

CORDEIRO, Z. M. J. Banana - Fitossanidade. Brasília: Embrapa Comunicação para Transferência de Tecnologia Brasília, 2000. 121 p. (Série Frutas do Brasil, 8).

FERREIRA, D. M. V.; CORDEIRO, Z. J. M.; MATOS, A. P. de. Sistema de pré-aviso para o controle da Sigatoka-amarela da bananeira no Recôncavo Baiano. Revista Brasileira de Fruticultura, Jaboticabal, n. 25, p. 429-431, 2003.

FHIA - FUNDACIÓN HONDUREÑA DE INVESTIGACIÓN AGRÍCOLA. Annual Report 2003-2004. 2004. Disponível em: $\quad<$ http://www.fhia.org.hn/dowloads/informes_anuales/ iainglesfhia2003-2004.pdf>. Acesso em: 22 maio 2012.

FIORAVANÇO, J. C.; PAIVA, M. C. Sigatoka-Negra da bananeira. Revista Brasileira de Agrociência, Pelotas, v. 11, n. 2, p. 135-141, 2005.

FOURÉ, E. Leaf spot disease of banana and plantain caused by Mycosphaerella musicola and $M$. fijiensis. In: INTERNATIONAL NETWORK FOR THE IMPROVEMENT OF BANANA AND PLANTAIN, HONDURAS. Proceedings... The improvement and testing of Musa: a global partnership. Montpellier: INIBAP, 1994. p. 47-53.

GANRY, J.; LAVILLE, E. Les cercosporioses du bananier et leurs traitment; evolutions des méthodes de traitment. I. Traitments fongicides 2. Avertissement. Fruits, Paris, v. 38, n. 1, p. 3-20, 1983. GANRY, J.; MEYER, J. P. La lutte controlée contre le cercospora aux Antilles. Bases climatiques de l'avertissement. Tecnique d'observation et numération de la maladie. Fruits, Paris, v. 27, n. 11, p. 767-774, 1972. 
GASPAROTTO, L.; PEREIRA, J. C. R.; HANADA, R. E. Situação atual da Sigatoka-negra no Brasil. In: POLTRONIERI, L. S.; VERZIGNASSI, J. R. (Ed.). Fitossanidade na Amazônia: inovações tecnológicas. Belém: Embrapa Amazônia Oriental, 2007. p. 37-52.

IBGE - INSTITUTO BRASILEIRO DE GEOGRAFIA E ESTATÍSTICA. Levantamento sistemático da produção agrícola. 2012. Disponível em: <http://www.ibge.gov.br/home/ estatistica/indicadores/agropecuaria/lspa/lspa_201203.pdf $>$. Acesso em: 15 maio 2012.

MDIC - MINISTÉRIO DO DESENVOLVIMENTO, INDÚSTRIA E COMÉRCIO EXTERIOR. Plano de desenvolvimento do arranjo produtivo fruticultura do norte de Minas Gerais. 2005. Disponível em: <http://www.mdic.gov.br/arquivos/ dwnl_1248287941.pdf>. Acesso em: 15 maio 2012.
MORAES, W. DA S.; PEREIRA, T. G.; CARNEIRO, O. L. G. A situação atual da sigatoka-negra no Sudeste brasileiro. In: SIMPÓSIO BRASILEIRO SOBRE BANANICULTURA, 7, 2010, Registro. Anais... Registro: SBF/APTA-SP/ABAVAR, 2010. p. $43-$ 58.

PÉREZ, L.V. Epidemiología de la Sigatoka-negra (Mycosphaerella fijiensis Morelet) em Cuba. I. Prognóstico bio-climático de los plátanos de los tratamientos de fungicidas en bananos (Musa acuminata AAA). Revista Mexicana de Fitopatologia, Sonora, v. 18 , n. 1 , p. $15-26,2000$.

TODA FRUTA. Frutas de A a Z, banana. 2006. Disponível em: $<$ http://www.todafruta.com.br>. Acesso em: 11 jun. 2006. 